\title{
The temperature dependence of the cross section for the energy pooling process $\mathrm{Na}(3 \mathrm{P})+\mathrm{Na}(3 \mathrm{P}) \rightarrow \mathrm{Na}(4 \mathrm{D})+\mathrm{Na}(3 \mathrm{~S})$
}

\author{
V Horvatic, M Movre and C Vadla \\ Institute of Physics, Bijenicka 46, HR-10000 Zagreb, Croatia
}

Received 28 April 1999, in final form 16 August 1999

\begin{abstract}
We report the measurements of the temperature dependence of the cross section $\sigma_{4 \mathrm{D}}$ for the energy pooling process $\mathrm{Na}(3 \mathrm{P})+\mathrm{Na}(3 \mathrm{P}) \rightarrow \mathrm{Na}(4 \mathrm{D})+\mathrm{Na}(3 \mathrm{~S})$. The latest two, as yet undisputed, results for $\sigma_{4 \mathrm{D}}$ obtained by different authors at $T=597 \mathrm{~K}$ and $T=483 \mathrm{~K}$ suggest that this cross section decreases with increasing $T$, which contradicts the theory and other experiments on similar processes. To resolve this controversy and to examine the temperature trend of the cross section, we have measured the $\sigma_{4 \mathrm{D}}$ in the temperature range $567-705 \mathrm{~K}$, covering the hightemperature region that has not yet been investigated experimentally. To determine $\sigma_{4 \mathrm{D}}$ we have excited sodium atoms in the quasistatic wing of the D1 line using a cw dye laser and measured the fluorescence intensity for the $4 \mathrm{D} \rightarrow 3 \mathrm{P}_{3 / 2}$ transition, relative to the intensity of the optically thin quasistatic wing of the $\mathrm{D} 2$ line. The spatial distribution of the number density of the sodium atoms in the $3 \mathrm{P}_{3 / 2}$ state and the sodium ground-state number density were measured too. The method used for the determination of the cross section is advantageous since it entirely circumvents the need to account for the radiation trapping of 3P level radiation, which was substantial under experimental conditions of the ground-state densities being $10^{14}-10^{16} \mathrm{~cm}^{-3}$. The measurements of the cross section $\sigma_{4 \mathrm{D}}$ in the investigated temperature range have shown that it increases as $\sim \exp (-\Delta E / k T)$. From the experiment we obtained $\Delta E=(608 \pm 95) \mathrm{cm}^{-1}$, which is in excellent agreement with the energy defect $\left(613 \mathrm{~cm}^{-1}\right)$ for the considered process, and in fair agreement with the values which follow from recent theoretical calculations.
\end{abstract}

\section{Introduction}

Investigations of collisional excitation energy transfer (EET) processes by means of laser spectroscopy contribute a great deal to our better understanding of collisional dynamics and interatomic potentials. Particularly numerous are investigations of various EET reactions (collisional transfer between fine-structure states, energy pooling and quenching) involving homonuclear and heteronuclear alkali systems. The alkali metals are very suitable to study experimentally because of their strong resonance absorption and the accessibility of their resonance lines to dye and diode lasers. Due to their simple hydrogen-like structure, alkalis are also convenient for theoretical investigations of interatomic interactions. Experimental and theoretical papers in this area published up to 1975 have been summarized by Krause (1975), while an overview of more recent work can be obtained by following the extensive list of references in Demtröder (1996).

Among alkalis, the sodium case has been so widely studied, both theoretically and experimentally, that it represents a kind of benchmark for the alkali dimers family. The present investigation is concerned with energy pooling process

$$
\mathrm{Na}(3 \mathrm{P})+\mathrm{Na}(3 \mathrm{P}) \rightarrow \mathrm{Na}(4 \mathrm{D})+\mathrm{Na}(3 \mathrm{~S})
$$


which was observed for the first time by Allegrini et al (1976). The first quantitative result for the cross section $\sigma_{4 \mathrm{D}}$ for this process was obtained by Krebs and Schearer (1980) who reported the value of $9 \times 10^{-18} \mathrm{~cm}^{2}$ at the temperature $T=670 \mathrm{~K}$, with the uncertainty of an order of magnitude. Soon after, Kushawaha and Levental (1982) measured the temperature dependence of the $\sigma_{4 \mathrm{D}}$ cross section in the temperature range $465-510 \mathrm{~K}$. They found the absolute value of $\sigma_{4 \mathrm{D}}$ to be $\approx 4.5 \times 10^{-20} \mathrm{~cm}^{2}$ and observed a slight increase in $\sigma_{4 \mathrm{D}}$ with increasing temperature, that followed $\sim \exp (-\Delta E / k T)$ dependence. The activation energy $\Delta E=968 \mathrm{~cm}^{-1}$, which is about 1.5 times higher than the energy defect $\left(613 \mathrm{~cm}^{-1}\right)$ for the considered process, was found. The following two experiments performed by Huennekens and Gallagher (1983) and Allegrini et al (1983) resolved a quandary about the order of magnitude of the $\sigma_{4 \mathrm{D}}$. These experiments solved the difficulties concerning radiation trapping effects and the determination of the spatial distribution and absolute value of the density of the excited atoms, which were not rigorously taken into account in the former two experiments, and yielded the cross section values $(46 \pm 16) \times 10^{-16} \mathrm{~cm}^{2}$ at $600 \mathrm{~K}$ (Huennekens and Gallagher 1983 ) and $(64 \pm 21) \times 10^{-16} \mathrm{~cm}^{2}$ (Allegrini et al 1983) at $483 \mathrm{~K}$. Here, both $\sigma_{4 \mathrm{D}}$ values are multiplied by a factor of two compared with the published ones, to take proper account of the number of atom pairs in a cell, as pointed out by Bezuglov et al (1987). However, the two experimental values appear contradictory regarding the temperature trend of the cross section. The considered energy pooling reaction is endothermic and should have a cross section smaller at $483 \mathrm{~K}$ than at $600 \mathrm{~K}$, while the two experimental results put together vary in the opposite sense, i.e. the cross section tends to decrease with increasing $T$, which cannot be the case. Unfortunately, considering the original papers alone, it is not easy to give preference to either of the two results.

Several theoretical calculations of the $\sigma_{4 \mathrm{D}}$ cross section are due to Kowalczyk (1984), Geltman (1989), Philipsen et al (1993) and Yurova et al (1994). Among them the calculations performed by Yurova et al (1994) should be considered most reliable since they are based on the accurate ab initio potential curves for the $\mathrm{Na}_{2}$ molecule (Magnier et al 1993, 1994), while the other authors used a very approximate estimation of the molecular potential curves. Regarding the temperature dependence all theoretical results predict a mild rise in the cross section value with increasing temperature. As for the absolute value, the computed cross sections are all systematically smaller than the latest two experimental values. The $\sigma_{4 \mathrm{D}}$ values of Kowalczyk (1984) and Philipsen et al (1993) are roughly one order of magnitude smaller, while those obtained by Geltman (1989) and Yurova et al (1994) are closest to the experiments, the differences amounting to a factor of three at most. Yurova et al (1994) have found the strong influence of the potentials on the magnitude of the cross section. Thereupon, they pointed out a necessity for a more elaborate theoretical model that would include fine-structure effects, which might help to improve agreement between measured and calculated cross sections. Also, it has been suggested that new experiments should check the energy dependence of the cross section.

Here, we report the measurements of the cross section for the considered process in the temperature range $567-705 \mathrm{~K}$. This range includes the temperature previously investigated by Huennekens and Gallagher (1983), and extends into the region of higher temperatures where no data (apart from the unreliable datum of Krebs and Schearer (1980)) are available as yet. With the method we used it was not possible to investigate (see section 3) the temperatures as low as in the experiment of Allegrini et al (1983). Nevertheless, the range explored in the present experiment is close enough to the temperatures previously investigated, so as to expect the corresponding $\sigma_{4 \mathrm{D}}$ values to be within the combined error bars of the previous results, thus enabling the reliability of the used method to be judged. We used cw laser radiation to excite sodium atoms to the $3 \mathrm{P}$ state, and a combination of the fluorescence and absorption 
measurements to determine the cross section. The relative populations of the $3 \mathrm{P}$ and $4 \mathrm{D}$ levels were determined by measuring the ratio of the corresponding fluorescence emissions, while the spatial distribution of the number density of the sodium atoms in the resonance state, as well as the sodium ground-state density were measured by the laser absorption method. The severe trapping of the sodium resonance radiation present at the investigated sodium densities, has no effect on our results since the method used (Vadla et al 1996) does not require knowledge about the effective lifetime of the $3 \mathrm{P}$ state. Thus, a frequent and often serious source of the uncertainty of the cross section results has been eliminated in the present experiment. Also, the cross section values are determined with an improved accuracy (roughly a factor of two better than in previous experiments). The cross section $\sigma_{4 \mathrm{D}}$ has been found to increase with the temperature following the $\exp (-\Delta E / k T)$ dependence. The obtained value $\Delta E=(608 \pm 95) \mathrm{cm}^{-1}$ agrees very well with the energy defect for the investigated energy pooling process. Our results show that the cross section measured by Huennekens and Gallagher (1983) is in excellent agreement with our experiment, while the value reported by Allegrini et al (1983) differs by a factor of two compared with the $\sigma_{4 \mathrm{D}}(T)$ dependence predicted by our measurements.

\section{Experimental approach}

\subsection{Measurements of the quantities needed for the cross section evaluation}

The determination of the cross section for energy pooling process (1) required measurements of the following quantities: the fluorescence intensity $I_{569}$ of the energy pooling line corresponding to the $4 \mathrm{D} \rightarrow 3 \mathrm{P}_{3 / 2}$ transition, the fluorescence intensity $I_{3 / 2}$ in the optically thin region of the quasistatic wing of the $\mathrm{D} 2$ line, the spatial distribution $N_{3 / 2}(r)$ of the number density of sodium atoms in the $3 \mathrm{P}_{3 / 2}$ state, and the sodium ground-state number density $N_{3 \mathrm{~S}}$. In the following we will describe how each of these quantities was measured.

The experimental arrangement is shown in figure 1 . The sodium metal was contained in a resistively heated, T-shaped stainless-steel heat-pipe oven with Pyrex glass windows. The heat pipe was filled with argon as a buffer gas. The heat pipe was not running in the heat-pipe mode. The sodium vapour was confined within the column of length $L=(7 \pm 1) \mathrm{cm}$. To determine $L$, a thin tungsten wire was built in along the heat-pipe axis (thermionic diode configuration), and the heat pipe was heated to several temperatures covering the investigated range. After each heating the wire was dismounted and inspected under the microscope. The segment which was attacked by alkali vapour as well as metal droplets deposited at the hot zone boundaries were clearly observable, enabling the determination of the metal vapour column length with the stated accuracy. No systematic variation of $L$ with the change of the temperature was observed.

The sodium atoms were excited in the quasistatic region of the red wing of the D1 line, typically at detunings $\Delta \lambda_{L} \approx 0.01-0.4 \mathrm{~nm}$ from the line centre. The laser radiation from a single-mode frequency-stabilized ring-dye laser (Spectra Physics model 380 D, dye: Rh6G), pumped by an argon-ion laser (Spectra Physics model 2020), was used for this purpose. The wavelength and the power of the dye-laser radiation were measured with a Burleigh wavemeter and Coherent power meter, respectively. The dye-laser beam was collimated and its central part, selected using a circular aperture of $4 \mathrm{~mm}$ in diameter, was shone through the heat-pipe. The total power of the dye-laser beam prior to hitting the heat-pipe entrance window was 90$120 \mathrm{~mW}$ and its distribution across the dye-laser beam cross section was nearly homogeneous. The fluorescence from the excitation zone was observed at right angles to the laser beam propagation direction and analysed using a $1 \mathrm{~m} \mathrm{McPherson} \mathrm{monochromator} \mathrm{supplied} \mathrm{with} \mathrm{the}$ 


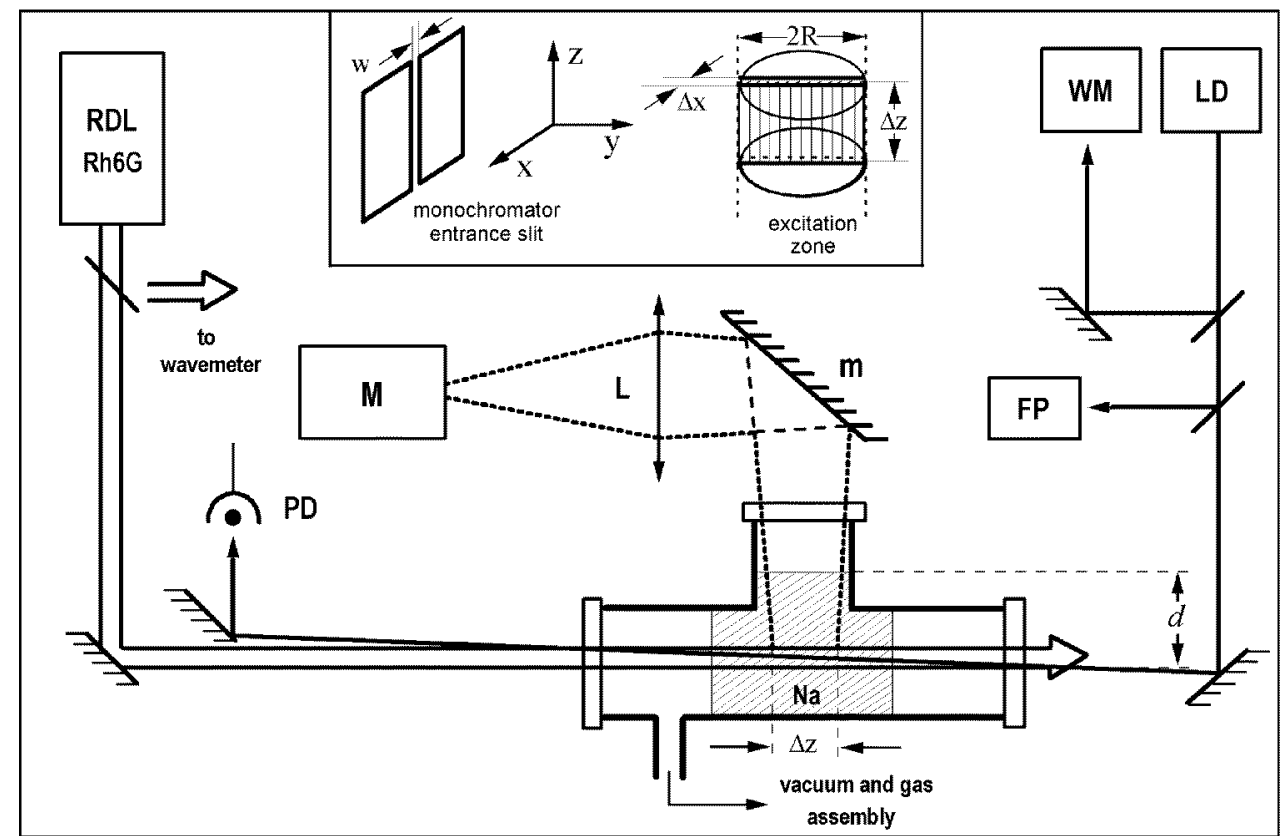

Figure 1. Experimental arrangement. RDL—ring-dye laser, $\mathrm{M}$-monochromator, L-lens, $\mathrm{m}-$ mirror, PD_photodiode, LD-laser diode, WM-wavemeter, FP-Fabry-Perot interferometer. The depicted inclination of the diode laser beams within the vapour column is largely exaggerated. The inset illustrates the orientation (after reflection on mirror $-\mathrm{m}$ ) of the observed excitation zone fragment $(2 R=0.4 \mathrm{~cm}, \Delta z \sim 1 \mathrm{~cm}, \Delta x=0.02 \mathrm{~cm} ; \Delta x$ is defined by the monochromator entrance slit width $w=0.01 \mathrm{~cm}$ and $1: 2$ imaging ratio) relative to the monochromator entrance slit.

RCA photomultiplier (type No C31034A). With the slit widths of $100 \mu \mathrm{m}$ the band pass of the monochromator was $0.1 \mathrm{~nm}$. The spectral response of the detection system was determined using a calibrated tungsten-ribbon lamp (type W2KGV22i).

The cylindrical fluorescence zone (length: $\sim 1 \mathrm{~cm}$ ) was imaged in 1:2 ratio onto the entrance slit of the monochromator as shown in figure 1 and its inset. The monochromator slits were parallel to the fluorescence zone axis (z-direction) and, with the aforementioned slit width, the observed fragment of the fluorescence zone was the central slab having the thickness $\Delta x=0.02 \mathrm{~cm}$.

At each particular temperature, the $3 \mathrm{P} \rightarrow 3 \mathrm{~S}$ and $4 \mathrm{D} \rightarrow 3 \mathrm{P}$ fluorescence, and the spatial distribution of the $3 \mathrm{P}_{3 / 2}$ population number density, were measured in a sequence with the pump laser kept locked at a chosen frequency detuned by $\Delta v_{L}$ in the red wing of the Na D1 line.

The fluorescence spectra of the resonant doublet and energy pooling lines were recorded by scanning the monochromator across the $3 \mathrm{P} \rightarrow 3 \mathrm{~S}$ and $4 \mathrm{D} \rightarrow 3 \mathrm{P}$ transitions.

The excited $3 \mathrm{P}_{3 / 2}$ population density was determined by measuring the absorption at the $3 \mathrm{P}_{3 / 2} \rightarrow 3 \mathrm{D}_{3 / 2,5 / 2}$ transition. The appropriate $819.7 \mathrm{~nm}$ radiation was supplied by a singlemode frequency-stabilized diode laser (Mitsubishi ML 2701, $\lambda=831 \mathrm{~nm}$ at $26{ }^{\circ} \mathrm{C}$, maximal power $8 \mathrm{~mW}$ ). The diode-laser beam (probe beam) was $0.5 \mathrm{~mm}$ in diameter and its power was reduced to $5 \mu \mathrm{W}$ using neutral density filters. It was aligned so as to pass through the centre of the excitation zone, counter-propagating to the pump beam. The absorption along 
the vapour column was measured by scanning the diode laser across the $3 \mathrm{P}_{3 / 2} \rightarrow 3 \mathrm{D}_{3 / 2,5 / 2}$ transition. The transmitted radiation was detected by a photodiode and the confocal FabryPerot interferometer (f.s.r. $2 \mathrm{GHz}$ ) was used to calibrate the dispersion of the spectrum. The measured spectra exhibited pure Doppler profile and the average number density $\bar{N}_{3 / 2}$ in the $\mathrm{Na}\left(3 \mathrm{P}_{3 / 2}\right)$ state along the path $L$ was determined from the peak absorption coefficient $k_{0}$ of the $3 \mathrm{P}_{3 / 2} \rightarrow 3 \mathrm{D}_{5 / 2}$ line according to the relation

$$
\bar{N}_{3 / 2}=\frac{k_{0}}{A} \frac{8 \pi^{3 / 2}}{\lambda^{3}} \frac{g_{1}}{g_{2}} \sqrt{\frac{2 k T}{M}}
$$

where $A=4.95 \times 10^{7} \mathrm{~s}^{-1}$ (Wiese et al 1969) is the spontaneous emission coefficient for the $3 \mathrm{D}_{5 / 2} \rightarrow 3 \mathrm{P}_{3 / 2}$ transition, $\lambda$ is the transition wavelength, $g_{1}$ and $g_{2}$ are the statistical weights of the $3 \mathrm{P}_{3 / 2}$ and $3 \mathrm{D}_{5 / 2}$ states, respectively, and $M$ is the atomic mass. The Doppler width of the $3 \mathrm{P}_{3 / 2} \rightarrow 3 \mathrm{D}_{3 / 2,5 / 2}$ lines at the experimental temperatures is $\sim 1.4 \mathrm{GHz}$ and the lines are separated by $\sim 1.3 \mathrm{GHz}$. Since the oscillator strength of the $3 \mathrm{P}_{3 / 2} \rightarrow 3 \mathrm{D}_{3 / 2}$ line is one order of magnitude smaller than the oscillator strength of the $3 \mathrm{P}_{3 / 2} \rightarrow 3 \mathrm{D}_{5 / 2}$ line, the contribution of the $3 \mathrm{P}_{3 / 2} \rightarrow 3 \mathrm{D}_{3 / 2}$ absorption to the peak absorption $k_{0}$ of the $3 \mathrm{P}_{3 / 2} \rightarrow 3 \mathrm{D}_{5 / 2}$ line is negligible.

The pump detunings $\Delta \lambda_{L}$ were chosen such that the attenuation of the pump laser intensity $I_{L}$ along optical path $L$ was always about $20 \%$, meaning that the $I_{L}$ decrease was practically linear in $z$. Consequently, the measured $\bar{N}_{3 / 2}$ describes well the actual $N_{3 / 2}$ at the centre of the vapour column, while the variation $( \pm 1.5 \%)$ of $N_{3 / 2}$ along the segment $\Delta z$ which was imaged onto the monochromator entrance slit can be safely ignored in further analysis.

The spatial distribution of the atoms in the $3 \mathrm{P}_{3 / 2}$ state is governed by the power profile of the pump laser beam, the excitation and the heat-pipe geometry, and is therefore radially symmetric. The $N_{3 / 2}(r)$ distribution was determined by translating the probe beam across the diameter ( $x$-direction) of the excitation zone and parallel with its axis, and measuring the corresponding absorption at the $3 \mathrm{P}_{3 / 2} \rightarrow 3 \mathrm{D}_{3 / 2,5 / 2}$ transition.

The Na ground-state number density $N_{3 \mathrm{~S}}$ was determined from $3 \mathrm{~S} \rightarrow 3 \mathrm{P}_{1 / 2}$ absorption measurements. For this purpose the power of the dye-laser beam was reduced using neutral density filters down to $10 \mu \mathrm{W}$. The dye laser was tuned to a series of discrete wavelengths across the red wing of the Na D1 line and the intensity transmitted through the sodium-vapour column of length $L$ was detected by a photodiode. The dispersion of the absorption spectrum was determined by the wavemeter readings. Applying the method described previously in Horvatic et al (1993) the sodium ground-state number density $N_{3 S}$ was determined from the set of measured values for the absorption coefficient $k(\Delta v)$, according to the relation which holds for the red wing of alkali D1 line (see the appendix, relation (A4)):

$$
N_{3 S}=\alpha \frac{\sqrt{k(\Delta v)}}{\sqrt{\mathcal{P}_{1 / 2}(Y)}} \Delta v
$$

where for the sodium $\alpha=1.17 \times 10^{5} \mathrm{~s} \mathrm{~cm}^{-5 / 2}, \Delta v$ is the detuning from the line centre (from this point on, all $\Delta v$ that appear are meant to be positive), $\mathcal{P}_{1 / 2}(Y)$ is a third-order polynomial in $Y=\Delta v / \Delta v_{\mathrm{fs}}\left(\Delta v_{\mathrm{fs}}=515.5 \mathrm{GHz}\right.$ is the fine-structure splitting of the $\mathrm{Na}(3 \mathrm{P})$ level $)$ given by relation (A2) in the appendix, and $k(\Delta v)$ is measured in $\mathrm{cm}^{-1}$.

Using relation (3) we found that in the experiment the sodium ground-state number density varied in the range from $2.2 \times 10^{14} \mathrm{~cm}^{-3}$ to $1.1 \times 10^{16} \mathrm{~cm}^{-3}$. The uncertainty of the $N_{3 \mathrm{~S}}$ is $\pm 10 \%$, and it mainly expresses the error in vapour-column length determination.

The temperature of the sodium vapour was determined in two ways. First, we used the measured values $N_{3 \mathrm{~S}}$ of the ground-state number density and read off the corresponding temperature from the vapour pressure curve (Nesmeyanov 1963). The temperatures obtained 
in this way were uncertain (disregarding the inaccuracy in the vapour pressure curve itself) as much as the error of $\pm 10 \%$ in the $N_{3 s}$ produced, i.e. typically less than $\pm 5 \mathrm{~K}$. Additionally, the $3 \mathrm{P}_{3 / 2} \rightarrow 4 \mathrm{D}_{3 / 2,5 / 2}$ absorption spectra were fitted to the sum of the two Doppler profiles having the same half-width and heights in the ratio of the corresponding oscillator strengths, while the temperature was left as a free parameter. By use of this fitting procedure the temperature of the vapour could be determined with the uncertainty of $\pm 15 \mathrm{~K}$. The absolute values of the temperature obtained in these two ways agreed within mutual error bars. The temperature interval covered by the measurements was found to be between $567 \mathrm{~K}$ and $705 \mathrm{~K}$.

These temperatures were used to obtain the Maxwellian mean relative velocity $\bar{v}$ of the colliding species. Accounting for the possible uncertainty in the vapour pressure curve we have estimated the error in $\bar{v}$ to be less than $\pm 2 \%$ (at the experimental temperatures an uncertainty of $\pm 20 \mathrm{~K}$ accounts for the stated error).

The total pressure in the heat pipe amounted to 1-2 Torr and was measured with a MKS Baratron manometer. The heat pipe was not running in a heat-pipe mode, therefore, the noble gas (argon) number density $N_{\mathrm{Ar}}$, in the region of the sodium-vapour column was obtained using the ideal-gas equation:

$$
p=N_{3 \mathrm{~s}} k T+N_{\mathrm{Ar}} k T
$$

where $k$ is the Boltzmann constant, $p$ is the total pressure measured by the manometer, while $T$ and $N_{3 S}$ are determined as described above.

\subsection{Method for the determination of the cross section from the experimental data}

The partial term diagram of sodium atom showing the energy levels involved in the determination of the cross section for the process (1) is displayed in figure 2. The sodium atoms in the $3 \mathrm{P}_{3 / 2}$ state were produced by cw laser excitation from the $\mathrm{Na}(3 \mathrm{~S})$ ground state. The initial $\mathrm{Na}(4 \mathrm{D})$ and $\mathrm{Na}(4 \mathrm{~F})$ populations were created through energy pooling collisions between two $\mathrm{Na}(3 \mathrm{P})$ atoms. Due to collisions with the ground-state $\mathrm{Na}$ and noble gas atoms the excitation energy transfer $\mathrm{Na}(4 \mathrm{D}) \underset{D}{\stackrel{C}{\rightleftarrows}} \mathrm{Na}(4 \mathrm{~F})$ between these two levels occurred at rates $C$ and $D$. The radiative relaxation of the $\mathrm{Na}(4 \mathrm{D})$ and $\mathrm{Na}(4 \mathrm{~F})$ levels, with spontaneous emission rates $A_{4 \mathrm{D}}=A_{4 \mathrm{D} \rightarrow 4 \mathrm{P}}+A_{4 \mathrm{D} \rightarrow 3 \mathrm{P}}$ and $A_{4 \mathrm{~F}}=A_{4 \mathrm{~F} \rightarrow 3 \mathrm{D}}$, respectively, involves the lower-lying $\mathrm{P}$ and $\mathrm{D}$ states as shown in figure 1 . Under the experimental conditions at hand, where the number density of the ground-state atoms is of the order of $10^{14}-10^{16} \mathrm{~cm}^{-3}$, the back-energy-pooling processes $\mathrm{Na}(4 \mathrm{D}, 4 \mathrm{~F})+\mathrm{Na}(3 \mathrm{~S}) \rightarrow \mathrm{Na}(3 \mathrm{P})+\mathrm{Na}(3 \mathrm{P})$ should not be neglected. Therefore, we take into consideration the rates $R_{4 \mathrm{D}}^{\text {back }}$ and $R_{4 \mathrm{~F}}^{\text {back }}$ corresponding to the $\mathrm{Na}(4 \mathrm{D})$ and $\mathrm{Na}(4 \mathrm{~F})$ collisional decay to the $\mathrm{Na}(3 \mathrm{P})$ level, respectively, induced by the ground-state $\mathrm{Na}$ atoms. The rate equations for the $r$-dependent, steady-state populations $N_{4 \mathrm{D}}(r)$ and $N_{4 \mathrm{~F}}(r)$ created under the described conditions, are the following:

$$
\begin{aligned}
& \frac{\mathrm{d} N_{4 \mathrm{D}}(r)}{\mathrm{d} t}=0=\frac{k_{4 \mathrm{D}}}{2}\left[N_{3 \mathrm{P}}(r)\right]^{2}+D N_{4 \mathrm{~F}}(r)-\left[A_{4 \mathrm{D}}+C+R_{4 \mathrm{D}}^{\text {back }}\right] N_{4 \mathrm{D}}(r) \\
& \frac{\mathrm{d} N_{4 \mathrm{~F}}(r)}{\mathrm{d} t}=0=\frac{k_{4 \mathrm{~F}}}{2}\left[N_{3 \mathrm{P}}(r)\right]^{2}+C N_{4 \mathrm{D}}(r)-\left[A_{4 \mathrm{~F}}+D+R_{4 \mathrm{~F}}^{\text {back }}\right] N_{4 \mathrm{~F}}(r) .
\end{aligned}
$$

Here $k_{4 \mathrm{D}}$ and $k_{4 \mathrm{~F}}$ label the rate coefficients for the process (1) and the analogous one involving the $\mathrm{Na}(4 \mathrm{~F})$ level, respectively. The solution to the system of equations (5), (6) reads

$$
N_{4 \mathrm{D}}(r)=k_{4 \mathrm{D}} \frac{1+\beta \mathcal{D}}{2 \mathcal{A}}\left[N_{3 \mathrm{P}}(r)\right]^{2}
$$

where the quantities $\mathcal{D}$ and $\mathcal{A}$ are given by

$$
\mathcal{D}=\frac{D}{A_{4 \mathrm{~F}}+D+R_{4 \mathrm{~F}}^{\text {back }}}
$$




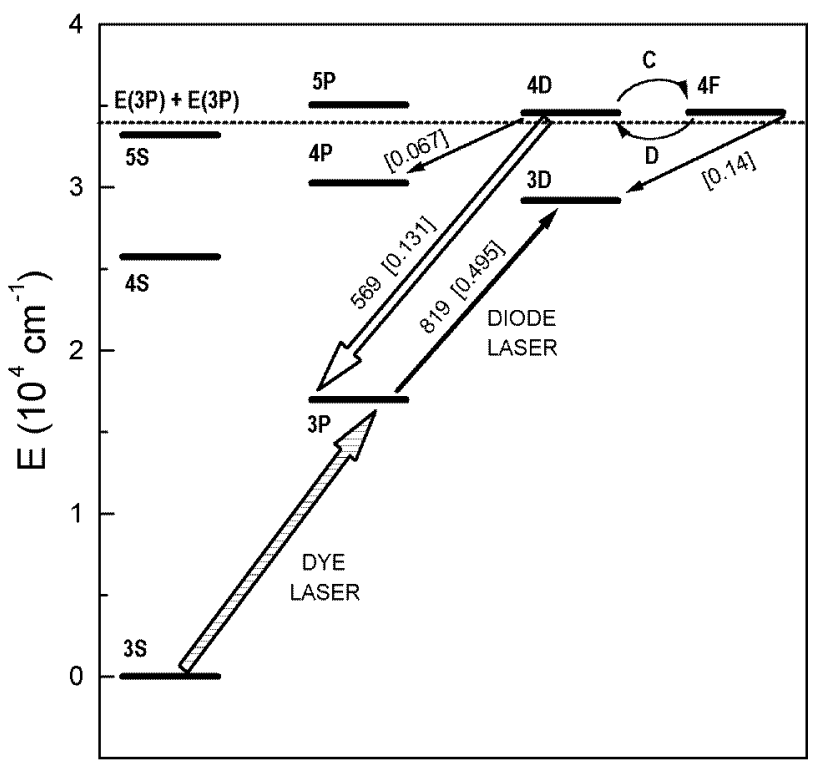

Figure 2. Partial term diagram of sodium. Energy levels and the processes involved in the determination of the rate coefficient $k_{4 \mathrm{D}}$ for the $\mathrm{Na}(3 \mathrm{P})+\mathrm{Na}(3 \mathrm{P}) \rightarrow \mathrm{Na}(4 \mathrm{D})+\mathrm{Na}(3 \mathrm{~S})$ energy pooling are depicted. Numerical values represent the wavelengths in nanometres and the spontaneous emission coefficients in $10^{8} \mathrm{~s}^{-1}$ (in brackets) for the relevant transitions. $C$ and $D$ are the rates for $4 \mathrm{D} \leftrightarrow 4 \mathrm{~F}$ mixing due to collisions with the ground-state sodium and argon atoms. To excite sodium atoms to the $3 \mathrm{P}$ state the dye laser was tuned to the red in the wing of the D1 line. The fluorescence was observed at the transitions indicated with hollow arrows. The population density in the $3 \mathrm{P}_{3 / 2}$ state was determined by measuring absorption at the $3 \mathrm{P}_{3 / 2} \rightarrow 3 \mathrm{D}_{3 / 2,5 / 2}$ transition.

and

$$
\mathcal{A}=A_{4 \mathrm{D}}+R_{4 \mathrm{D}}^{\mathrm{back}}+C(1-\mathcal{D})
$$

while $k_{4 \mathrm{~F}}$ is expressed as $k_{4 \mathrm{~F}}=\beta k_{4 \mathrm{D}}$. There are several values of $\beta$ that can be found in the literature, experimental as well as theoretical. In evaluation of the results we have used the experimentally determined value $\beta=0.55 \pm 0.05$, reported by Davidson et al (1986). The choice of $\beta$ and its influence on the determination of the $k_{4 \mathrm{D}}$ will be explained with the discussion of the results. The values $A_{4 \mathrm{D}}=1.98 \times 10^{7} \mathrm{~s}^{-1}$ and $A_{4 \mathrm{~F}}=1.4 \times 10^{7} \mathrm{~s}^{-1}$ are taken from Wiese et al (1969).

The collisional mixing rates $C$ and $D$ are defined as a product $N \sigma \bar{v}$, where $N$ is the number density of the perturbers inducing the 4D $\leftrightarrow 4 \mathrm{~F}$ mixing, $\sigma$ is the corresponding cross section and $\bar{v}$ is the mean Maxwellian relative velocity of the colliding species. The rate $C$ was calculated according to the expression

$$
C=N_{3 \mathrm{~S}} \sigma_{\mathrm{Na}-\mathrm{Na}} \bar{v}_{\mathrm{Na}-\mathrm{Na}}+N_{\mathrm{Ar}} \sigma_{\mathrm{Na}-\mathrm{Ar}} \bar{v}_{\mathrm{Na}-\mathrm{Ar}}
$$

which takes into account that the heat pipe was not running in the heat-pipe mode, so that $C$ includes contributions due to collisions between $\mathrm{Na}$ (4D) atoms and both sodium and argon ground-state atoms. The values for $N_{\mathrm{Na}}, N_{\mathrm{Ar}}$ and $\bar{v}$ were determined in the experiment as described previously, while for the cross sections for the $4 \mathrm{D} \rightarrow 4 \mathrm{~F}$ excitation transfer we used the values $\sigma_{\mathrm{Na}-\mathrm{Na}}=(410 \pm 50) \times 10^{-16} \mathrm{~cm}^{2}, \sigma_{\mathrm{Na}-\mathrm{Ar}}=(28 \pm 6) \times 10^{-16} \mathrm{~cm}^{2}$ measured by Horvatic et al (1997). The rate $D$ was calculated applying the principle of detailed balance, which for the temperatures in the experiment yields the ratio $C / D=1.3$. 
The back-pooling rates $R_{4 \mathrm{D}}^{\text {back }}$ and $R_{4 \mathrm{~F}}^{\text {back }}$ are expressed as $k_{j}^{\text {back }} N_{3 \mathrm{~S}}(j=4 \mathrm{D}, 4 \mathrm{~F})$. The rate coefficients for the forward- and back-pooling processes are related through the principle of detailed balance, i.e. $k_{j}=\left(g_{j} k_{j}^{\text {back }} / g_{i}\right) \times \exp \left(-\Delta E_{j} / k T\right)$, where $g_{i}$ and $g_{j}$ are the statistical weights of the initial $(3 \mathrm{P}+3 \mathrm{P})$ and final $(j+3 \mathrm{~S})$ state, respectively $\left(g_{i}=9, g_{4 \mathrm{D}}=10, g_{4 \mathrm{~F}}=14\right)$ and $\Delta E_{j}$ is the energy defect for the considered process $\left(\Delta E_{4 \mathrm{D}}=613.5 \mathrm{~cm}^{-1}, \Delta E_{4 \mathrm{~F}}=653.3 \mathrm{~cm}^{-1}\right)$. Consequently, the back-pooling rates acquire the form $R_{j}^{\text {back }}=e_{j} k_{j} N_{3 \mathrm{~S}}$, where $e_{j}=\left(g_{i} / g_{j}\right) \times \exp \left(\Delta E_{j} / k T\right)$.

The rate equations (5), (6) are valid for any $r$. If we apply these equations to the centre of the excitation zone $(r=0)$, and take into account all the above explanations, from relation (7) we obtain the following expression for the rate coefficient $k_{4 \mathrm{D}}$ :

$$
k_{4 \mathrm{D}}=\frac{2}{(1+\eta)^{2}} \frac{N_{4 \mathrm{D}}^{c}}{\left(N_{3 / 2}^{c}\right)^{2}} \frac{a_{0}+a_{1} k_{4 \mathrm{D}}+a_{2} k_{4 \mathrm{D}}^{2}}{b_{0}+b_{1} k_{4 \mathrm{D}}}
$$

where $\eta=N_{1 / 2}^{c} / N_{3 / 2}^{c}, N_{4 \mathrm{D}}^{c}, N_{3 / 2}^{c}$ and $N_{1 / 2}^{c}$ label the population densities measured at the excitation zone axis, while the polynomial coefficients appearing in the right-hand side of the above equation are defined as follows:

$$
\begin{aligned}
& a_{0}=A_{4 \mathrm{D}} A_{4 \mathrm{~F}}+C\left(A_{4 \mathrm{~F}}+0.77 A_{4 \mathrm{D}}\right) \\
& a_{1}=N_{3 \mathrm{~S}}\left[e_{4 \mathrm{D}}\left(A_{4 \mathrm{~F}}+0.77 C\right)+\beta e_{4 \mathrm{~F}}\left(A_{4 \mathrm{D}}+C\right)\right] \\
& a_{2}=\beta e_{4 \mathrm{D}} e_{4 \mathrm{~F}} N_{3 \mathrm{~S}}^{2} \\
& b_{0}=A_{4 \mathrm{~F}}+0.77 C(1+\beta) \\
& b_{1}=\beta e_{4 \mathrm{~F}} N_{3 \mathrm{~S}} .
\end{aligned}
$$

Since at each experimental temperature all quantities needed for the evaluation of the polynomial coefficients in equation (10) are known and the ratio $N_{4 \mathrm{D}}^{c} /\left(N_{3 / 2}^{c}\right)^{2}$ is determined in the experiment, the rate coefficient $k_{4 \mathrm{D}}$ is obtained as a solution to the equation $k_{4 \mathrm{D}}=f\left(k_{4 \mathrm{D}}\right)$ defined by relation (10).

The $N_{4 \mathrm{D}}^{c} /\left(N_{3 / 2}^{c}\right)^{2}$ ratio was determined using the method that has been employed and described previously (Vadla et al 1996, Movre et al 1999), but for the reader's convenience the procedure will be briefly outlined below.

The absorption coefficient $k(v)$ and the spectral emission coefficient $A^{v}(v)$ are related through the expression

$$
k(v)=N \frac{g_{2}}{g_{1}} \frac{\lambda^{2}}{8 \pi} A^{v}(v)
$$

where $g_{1}$ and $g_{2}$ are the statistical weights of the ground and excited state, respectively, $\lambda$ is the wavelength of the transition, and $N$ is the ground-state number density.

The absorption coefficient in the outer quasistatic wings of the self-broadened alkali first resonance doublets is reliably expressed by relations (A4) and (A5) (see the appendix for details). By combining equations (12) and (A5) we arrive at the following expression for the spectral emission coefficient $A_{3 / 2}^{v}(v)$ :

$$
A_{3 / 2}^{v}(v)=\frac{\pi e^{4} \nu_{3 / 2} f_{3 / 2}^{2}}{3 m^{2} c^{3}} \frac{g_{1}}{g_{2}} N_{3 S} \frac{\mathcal{P}_{3 / 2}(Y)}{(\Delta v)^{2}}=0.263 \times N_{3 S} \frac{\mathcal{P}_{3 / 2}(Y)}{(\Delta v)^{2}}
$$

which is applicable for the blue wing of the sodium D2 line and holds for the detunings $\Delta v$ in the wing not greater than $3 \times \Delta v_{\mathrm{fs}}$. In relation $(13), \mathcal{P}_{3 / 2}(Y)$ is the fourth-order polynomial in $Y$ given by equation (A3).

The spectral intensity of the optically thin quasistatic blue wing of the sodium D2 line emitted at the detuning $\Delta v$ from the infinitesimal volume $\mathrm{d} V$ is given by

$$
\mathrm{d} I_{3 / 2}^{v}(\Delta v)=h v_{3 / 2} A_{3 / 2}^{v} \mathrm{~d} v N_{3 / 2}(\Delta v, x, y, z) \mathrm{d} V .
$$


The intensity of the emitted radiation is spectrally integrated over the frequencies within the band pass $\delta v$ of the detection system. If the band pass is small compared with the detuning $\Delta v=v_{3 / 2}-v$ from the line centre, the spectral emission coefficient (relation (13)) can be regarded as unchanged within the interval $(v, v+\delta v)$. In equation (14) the $N_{3 / 2}(\Delta v, x, y, z)$ represents a small portion of the atoms being in the $3 \mathrm{P}_{3 / 2}$ state and radiating in the quasistatic wing at the frequency $v_{3 / 2}-\Delta v$, i.e. $N_{3 / 2}(\Delta v, x, y, z) \propto N_{3 / 2}(x, y, z) \exp (-h \Delta v / k T)$, where $N_{3 / 2}(x, y, z)$ denotes the number density of the atoms radiating in the centre of the line. The $N_{3 / 2}(x, y, z)$ population density is independent of $z$ (as explained in section 2.1) and it can also be regarded as independent of $x$ along the segment $\Delta x(\Delta x \ll 2 R)$ (see figure 6). In this case, only the inhomogeneity in the direction of observation ( $y$-direction) is relevant, and the emerging fluorescence intensity acquires the form

$$
I_{3 / 2}(\Delta v) \propto h v_{3 / 2} A_{3 / 2}^{v} \delta v N_{3 / 2}^{\mathrm{c}} \exp (-h \Delta v / k T) \int_{\Delta V} \mathcal{F}_{3 / 2}(y) \mathrm{d} y .
$$

Here, we have expressed $N_{3 / 2}(y)=N_{3 / 2}^{\mathrm{c}} \mathcal{F}_{3 / 2}(y)$, where $N_{3 / 2}^{\mathrm{c}}$ denotes population density at the excitation zone axis and $\mathcal{F}_{3 / 2}(y)$ is the radial distribution function normalized to unity at $y=0$. The fluorescence intensity of the optically thin line arising in the $4 \mathrm{D} \rightarrow 3 \mathrm{P}_{3 / 2}$ transition, observed by the monochromator having the band pass large $(0.1 \mathrm{~nm}$ in the present case) in comparison with the width of the line, is spectrally integrated over the frequencies within the whole line profile. Therefore, the total fluorescence intensity of the $569 \mathrm{~nm}$ line emerging from the observation volume (see the inset in figure 1) can be expressed as

$$
I_{569} \propto h v_{569} A_{569} \int_{\Delta V} N_{4 \mathrm{D}}(x, y, z) \mathrm{d} x \mathrm{~d} y \mathrm{~d} z
$$

where $A_{569}=0.4 A_{4 \mathrm{D}_{3 / 2} \rightarrow 3 \mathrm{P}_{3 / 2}}+0.6 A_{4 \mathrm{D}_{5 / 2} \rightarrow 3 \mathrm{P}_{3 / 2}}=8.74 \times 10^{6} \mathrm{~s}^{-1}$ denotes the total spontaneous emission coefficient for the $4 \mathrm{D} \rightarrow 3 \mathrm{P}_{3 / 2}$ transition. Here, we have anticipated the experimental finding that 4D fine-structure levels are populated in their statistical ratio. The values $A_{4 \mathrm{D}_{3 / 2} \rightarrow 3 \mathrm{P}_{3 / 2}}=2.2 \times 10^{6} \mathrm{~s}^{-1}$ and $A_{4 \mathrm{D}_{5 / 2} \rightarrow 3 \mathrm{P}_{3 / 2}}=1.31 \times 10^{7} \mathrm{~s}^{-1}$ are taken from Wiese et al (1969).

The $N_{4 \mathrm{D}}$ and $N_{3 / 2}$ are related through equation (7), and since $N_{3 / 2}$ is only $y$-dependent, $N_{4 \mathrm{D}}$ is also only a function of $y$. Analogously as before, we express $N_{4 \mathrm{D}}(y)=N_{4 \mathrm{D}}^{\mathrm{c}} \mathcal{F}_{4 \mathrm{D}}(y)$, and obtain the following expression for the $I_{569}$ fluorescence intensity:

$$
I_{569} \propto h v_{569} A_{569} N_{4 \mathrm{D}}^{\mathrm{c}} \int_{\Delta V} \mathcal{F}_{4 \mathrm{D}}(y) \mathrm{d} y .
$$

By combination of equations (10), (13), (15) and (17) we obtain the final expression for $k_{4 \mathrm{D}}$ :

$k_{4 \mathrm{D}}=\frac{8.446 \times 10^{3}}{(1+\eta)^{2}} \frac{I_{569}^{\text {meas }}}{I_{3 / 2}^{\text {meas }}(\Delta v)} \frac{N_{3 \mathrm{~S}}}{N_{3 \mathrm{P}_{3 / 2}}^{\mathrm{c}}} \frac{a_{0}+a_{1} k_{4 \mathrm{D}}+a_{2} k_{4 \mathrm{D}}^{2}}{b_{0}+b_{1} k_{4 \mathrm{D}}} \frac{\mathcal{P}_{3 / 2}(\Delta v)}{(\Delta v)^{2}} \mathrm{e}^{-h \Delta v / k T}$.

Here, we have substituted numerical values for all known quantities that appear, and have taken into account the fact that the intensities $I_{569}$ and $I_{3 / 2}$ are related to the measured ones by $I_{569} / I_{3 / 2}=I_{569}^{\text {meas }} \varepsilon_{3 / 2} / I_{3 / 2}^{\text {meas }} \varepsilon_{569}=1.17 \times I_{569}^{\text {meas }} / I_{3 / 2}^{\text {meas }}$, where $\varepsilon_{569}$ and $\varepsilon_{3 / 2}$ denote the spectral responses of the detection system at the relevant wavelengths. Also, we have accounted for the fact that $\mathcal{F}_{4 \mathrm{D}}(r)=\mathcal{F}_{3 / 2}^{2}(r)$ (see Vadla et al $(1996,1998)$ and the argumentation in the following section), and since $\mathcal{F}_{3 / 2}(r)$ is found to exhibit Gaussian shape the ratio of the integrals of the $4 \mathrm{D}$ and $3 \mathrm{P}_{3 / 2}$ distributions amounts to 0.7 . There is no need to correct either of the measured fluorescence intensities for the absorption on the optical path $d$ (see figure 1). Namely, the $3 \mathrm{~S}_{1 / 2} \rightarrow 3 \mathrm{P}_{3 / 2}$ absorption (measured on the optical path $L \approx 4 d$ ) was not detectable at wing detunings $\Delta v(\Delta \lambda \geqslant 0.6 \mathrm{~nm})$ appearing in equation (18). With actual $\mathcal{F}_{3 / 2}(r)$ (see figure 6) and the highest $N_{3 / 2}^{\mathrm{c}}$ ever realized in the present experiment $\left(1.4 \times 10^{11} \mathrm{~cm}^{-3}\right)$, the correction 


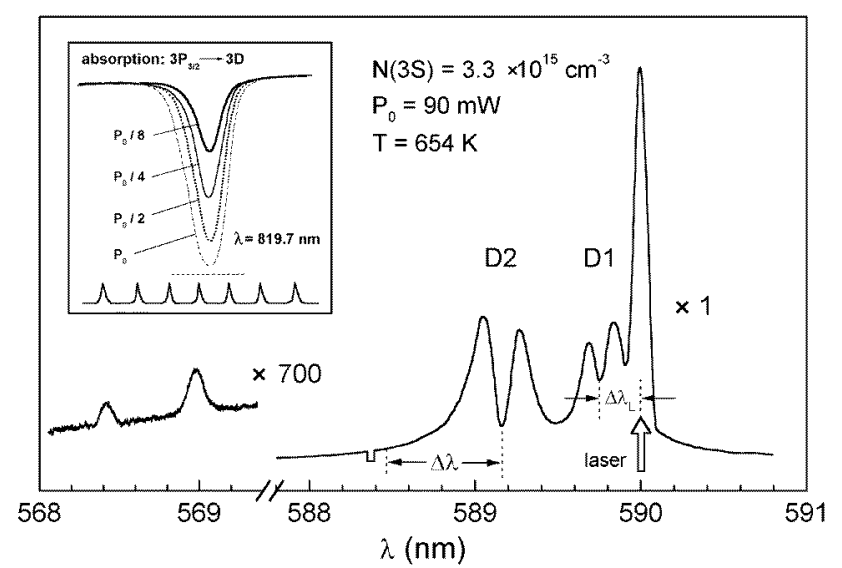

Figure 3. The fluorescence spectra measured by scanning the monochromator, while the dye laser was locked at the wavelength detuned by $\Delta \lambda_{L}$ to the red from the centre of the Na D1 line; $\Delta \lambda(\Delta \lambda \gtrsim 0.5 \mathrm{~nm})$ denotes detunings at which the D2 blue wing fluorescence intensities, used in the evaluation of the results, were taken. The presented fluorescence spectra were measured with full pump power applied $\left(P_{0}=90 \mathrm{~mW}\right)$, at the sodium ground-state number density $N_{3 \mathrm{~S}}=3.3 \times 10^{15} \mathrm{~cm}^{-3}$ and $T=654 \mathrm{~K}$. The displayed spectra are not corrected for the spectral response of the system. The inset shows the $3 \mathrm{P}_{3 / 2} \rightarrow 3 \mathrm{D}$ absorption spectra measured for different applied pump powers. The number density in the $3 \mathrm{P}_{3 / 2}$ state created with the pump power $P_{0} / 8$ was $5 \times 10^{9} \mathrm{~cm}^{-3}$, which yielded the population of $4 \times 10^{10} \mathrm{~cm}^{-3}$ corresponding to the $P_{0}$. The spectra are calibrated by transmission peaks of the Fabry-Perot interferometer that are separated by $2 \mathrm{GHz}$.

for the absorption of the $568.9 \mathrm{~nm}$ radiation at the optical path $r$ corresponding to the radial dimension of the fluorescence zone $(-0.3 \mathrm{~cm} \lesssim r \lesssim 0.3 \mathrm{~cm})$, amounts to $2 \%$. Since this correction is smaller than the uncertainty of the fluorescence intensity readings $( \pm 5 \%)$, it was neglected in the evaluation of the results.

\section{Measurements and results}

Figure 3 shows typical spectra measured while sodium atoms were excited by laser radiation at full pump power. The frequency of the dye laser was kept locked at the frequency detuned by $\Delta v_{L}$ in the red wing of the D1 line, and the fluorescence intensities of the sodium resonance doublet and the $568.9 \mathrm{~nm}$ and $568.4 \mathrm{~nm}$ energy pooling lines $\left(4 \mathrm{D}_{3 / 2,5 / 2} \rightarrow 3 \mathrm{P}_{3 / 2}\right.$ and $4 \mathrm{D}_{3 / 2} \rightarrow 3 \mathrm{P}_{1 / 2}$ transitions) were recorded by scanning the monochromator. The monitored fluorescence emerged from the thin slab in the centre of the excitation zone (see the inset in figure 1). Note that the intensities of the $568.9 \mathrm{~nm}$ and $568.4 \mathrm{~nm}$ lines are in the ratio 2:1, reflecting complete mixing of the 4D fine-structure sublevels, as assumed in the evaluation of equation (18). The inset in figure 3 shows the absorption measured at the $3 \mathrm{P}_{3 / 2} \rightarrow 3 \mathrm{D}$ transition used for determination of the population density $N_{3 / 2}^{\mathrm{c}}$ in the $3 \mathrm{P}_{3 / 2}$ state. When full pump power $P_{0}$ was applied, the $819.7 \mathrm{~nm}$ line exhibited total, or near-total absorption. To obtain an optically thin absorption spectrum appropriate for the $N_{3 / 2}^{\mathrm{c}}$ determination, the power $P_{0}$ was halved several times using neutral density filters. Unfortunately, with the reduced pump power the energy pooling line was not measurable. Therefore, we determined the $N_{3 / 2}^{\mathrm{c}}$ that corresponded to the $P_{0}$ in the following way. The population density $\tilde{N}_{3 / 2}^{\mathrm{c}}$, created with the lowest used pump power $\tilde{P}$, was obtained from the corresponding peak absorption coefficient 


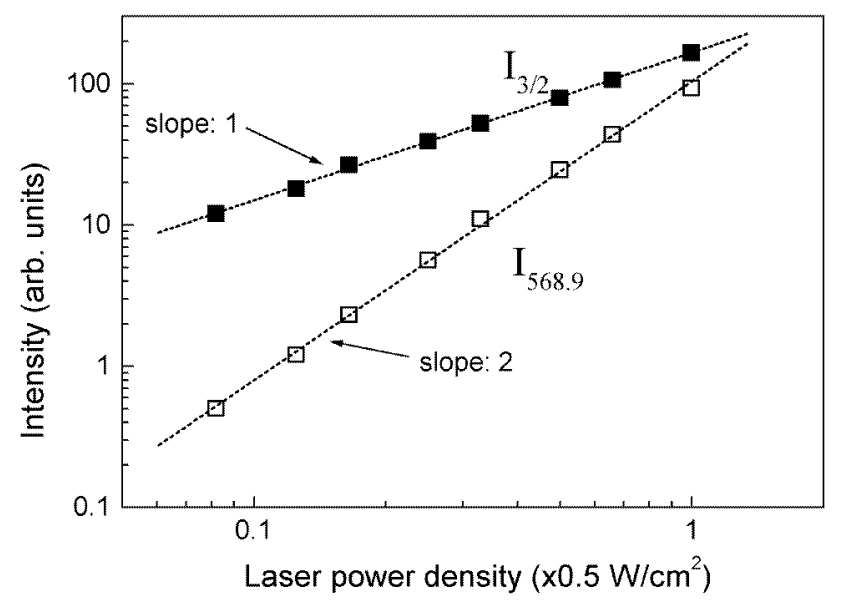

Figure 4. The fluorescence intensity $I_{3 / 2}$ of the optically thin blue wing of the Na D2 line monitored at the fixed detuning from the line centre and the intensity $I_{569}$ of the energy pooling line at $568.9 \mathrm{~nm}\left(4 \mathrm{D} \rightarrow 3 \mathrm{P}_{3 / 2}\right.$ transition) measured in dependence on the applied pump power. All relevant experimental conditions (temperature, argon pressure, pump frequency $v_{L}$ ) were kept constant and the pump power was reduced in steps using neutral density filters. The data show that the $\mathrm{D} 2$ wing intensity, which in the conditions at hand reflects $3 \mathrm{P}_{3 / 2}$ population density, scales in linear proportion with the applied pump power. The intensity of the energy pooling line exhibits $I_{569} \propto I_{3 / 2}^{2}$ behaviour in the whole range of the applied pump powers, confirming that the full pump power caused no detectable trapping of the $568.9 \mathrm{~nm}$ radiation.

of the $819.7 \mathrm{~nm}$ line, and the population density $N_{3 / 2}^{\mathrm{c}}$ realized with the full pump power was calculated according to $N_{3 / 2}^{\mathrm{c}}=\tilde{N}_{3 / 2}^{\mathrm{c}} P_{0} / \tilde{P}$. We have verified that $N_{3 / 2}^{\mathrm{c}}$ scales with the pump power by measuring the fluorescence wing intensity at certain fixed detuning in the blue wing of the D2 line as a function of the applied pump power. The result is shown in figure 4 where it can be seen that as the pump power reduces, the wing intensity $I_{3 / 2}$ reduces by the same factor. This confirms that $N_{3 / 2}^{\mathrm{c}}$ varies proportionally with the applied power as well, since in the conditions of constant ground-state number density, the wing intensity $I_{3 / 2}$ reflects the population density in the $3 \mathrm{P}_{3 / 2}$ state. Therefore, it is justified to determine the $N_{3 / 2}^{\mathrm{c}}$ in the previous manner. Typical number densities in the $3 \mathrm{P}_{3 / 2}$ state created with the full pump power varied between $4 \times 10^{10} \mathrm{~cm}^{-3}$ and $1.4 \times 10^{11} \mathrm{~cm}^{-3}$, depending on the $P_{0}$ and the pump detuning $\Delta v_{L}$ used. To check whether such $N_{3 / 2}^{\mathrm{c}}$ densities caused any trapping of the $4 \mathrm{D} \rightarrow 3 \mathrm{P}_{3 / 2}$ fluorescence, the intensity $I_{569}$ of the energy pooling line at $568.9 \mathrm{~nm}$ was simultaneously measured in dependence on the applied pump power. In the absence of trapping the intensity $I_{569}$ should vary as $I_{569} \propto\left(N_{3 / 2}^{\mathrm{c}}\right)^{2}$ (see relations (7) and (17)), or in terms of the intensity, $I_{569} \propto I_{3 / 2}^{2}$. If any trapping was present, the increase of the $I_{569}$ with increasing $N_{3 / 2}^{\mathrm{c}}$ would be weaker than stated, due to decreased spontaneous emission rate $A_{569}$. The measured $I_{3 / 2}$ versus $P$ and $I_{569}$ versus $P$ dependences are presented in a $\log -\log$ plot in figure 4 , in which both quantities exhibit linear increase with increased pump power in the whole range of the applied powers. The slopes of the corresponding straight lines fitted through the data are 1 and 2, respectively, as they should be in the absence of trapping of the $568.9 \mathrm{~nm}$ radiation.

The reliability of the determination of the rate coefficient $k_{4 \mathrm{D}}$ according to equation (18) depends on the extent to which the emission profile of the sodium D2 line in the quasistatic region of its blue wing is indeed reproduced by the theoretical absorption profile that we used. The evidence is displayed in figure 5 , where the measured fluorescence wing intensity 


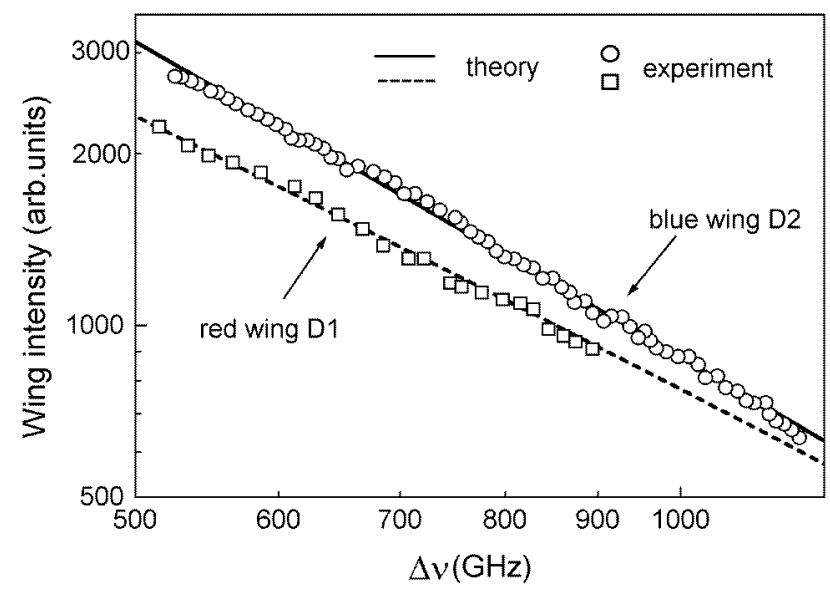

Figure 5. The fluorescence intensities (corrected for corresponding Boltzmann factors to enable comparison with theoretical absorption profiles-see equations (12), (15), (A4) and (A5)) of the outer wings of the sodium resonance doublet in dependence on the detuning from the respective line centre: $\mathrm{O}$-blue wing of the D2 line, $\square$-red wing of the D1 line. Theoretical absorption profiles (normalized by the measured wing intensity values at $\Delta v=800 \mathrm{GHz}(\Delta \lambda=0.9 \mathrm{~nm})$ ) are represented by full and dashed lines.

(corrected for the Boltzmann factor) as a function of the detuning $\Delta v$ from the centre of the D2 line is plotted together with the theoretical quasistatic absorption profile. It is evident that the agreement between the two is most satisfying. The results for the red wing of the D1 line are also included in the figure.

At the actual ground-state sodium densities $\sim 5 \times 10^{15} \mathrm{~cm}^{-3}$, the $3 \mathrm{P}_{1 / 2} \leftrightarrow 3 \mathrm{P}_{3 / 2}$ mixing rate due to collisions with ground-state sodium and argon atoms is faster than the effective spontaneous emission rate for both $3 \mathrm{P}_{1 / 2}$ and $3 \mathrm{P}_{3 / 2}$ states, and the ratio $N_{3 / 2} / N_{1 / 2}=\eta^{-1}$ approaches the thermodynamic value of $2 \exp (-\Delta E / k T)=1.92(T=640 \mathrm{~K})$. In the evaluation of the results we used the value of $\eta$ determined from the experiment. Using equation (18) and the analogous one for the $I_{1 / 2}(\Delta v)$, from the measured fluorescence intensities in the outer wings of the $\mathrm{D}$ lines (the spatial distributions of the $3 \mathrm{P}_{1 / 2}$ and $3 \mathrm{P}_{3 / 2}$ atoms were found to be the same) we obtained the value $\eta^{-1}=1.67$, rather than the thermodynamic ratio of 1.92 . A similar effect was observed earlier by Davidson et al (1986). They worked at sodium groundstate densities of $\sim 3 \times 10^{14} \mathrm{~cm}^{-3}$ and, when pumping the $3 \mathrm{P}_{1 / 2}$ state, obtained $\eta^{-1}=1.50$ instead of the thermodynamic ratio of 1.92 corresponding to their experimental temperature $(T=640 \mathrm{~K})$.

The spatial distribution of the sodium number density in the $3 \mathrm{P}_{3 / 2}$ state was measured as described in section 2 , and the result is displayed in figure 6 , where the normalized distribution $\mathcal{F}_{3 / 2}(r)$ is shown. The $3 \mathrm{P}_{3 / 2}$ atoms are well confined to the excitation zone. Although this may seem unusual in the conditions of severe trapping of the resonance radiation, the effect of the spatial distribution shrinkage at high ground-state densities has already been observed (Scholz et al 1996). (a) The normalized distribution of the $4 \mathrm{D}$ atoms behaves like $\mathcal{F}_{4 \mathrm{D}}(r)=\mathcal{F}_{3 / 2}^{2}(r)$. (b) This behaviour of the normalized distribution of the 4D atoms is the consequence of the $N_{4 \mathrm{D}}^{\mathrm{c}} \sim\left(N_{3 / 2}^{\mathrm{c}}\right)^{2}$. (c) The evidence for the $N_{4 \mathrm{D}}^{\mathrm{c}} \sim\left(N_{3 / 2}^{\mathrm{c}}\right)^{2}$ proportionality was given above. The $\mathcal{F}_{3 / 2}(r)$ exhibits the Gaussian shape which leads to the ratio of the integrals of the 4D and $3 \mathrm{P}_{3 / 2}$ distributions of 0.7 , as mentioned previously in section 2.2 .

The overall error bar of the $\sigma_{4 \mathrm{D}}$ data includes contributions due to the uncertainties in 


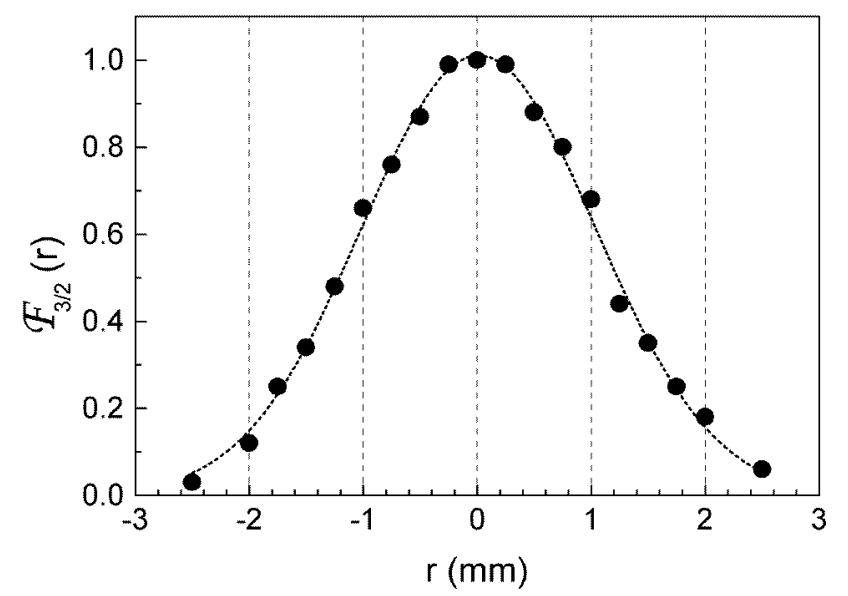

Figure 6. The spatial distribution $\mathcal{F}_{3 / 2}(r)$ of the sodium atoms excited to the $3 \mathrm{P}_{3 / 2}$ state, normalized to unity at $r=0$. The dashed curve is obtained by fitting the data to the Gaussian profile.

the experimental quantities $I_{3 / 2}, I_{569}, N_{3 \mathrm{~S}}, N_{3 / 2}^{\mathrm{c}}$, and the quantities taken from the literature and used in the evaluation $\left(\sigma_{\mathrm{Na}-\mathrm{Na}}\right.$ and $\sigma_{\mathrm{Na}-\mathrm{Ar}}$ in relation (9)). The variation of the $\sigma_{\mathrm{Na}-\mathrm{Na}}$ and $\sigma_{\mathrm{Na}-\mathrm{Ar}}$ within their error bars produces the uncertainty in the rate $C$ of $\pm 15 \%$ at most. Such an inaccuracy in $C$ affects the solution of equation (18) by less than $\pm 1 \%$, since the term involving the ratio of the polynomials in $k_{4 \mathrm{D}}$ alters less than $2 \%$ in the interval of the $k_{4 \mathrm{D}}$ values as wide as $10^{-11} \mathrm{~cm}^{3} \mathrm{~s}^{-1} \leqslant k_{4 \mathrm{D}} \leqslant 10^{-9} \mathrm{~cm}^{3} \mathrm{~s}^{-1}$ (our measured $k_{4 \mathrm{D}}$ values are $\sim 10^{-10} \mathrm{~cm}^{3} \mathrm{~s}^{-1}$ ). Therefore, the contribution to the overall $k_{4 \mathrm{D}}$ error bar from this source can be safely disregarded. As mentioned previously, the uncertainties of the number densities $N_{3 S}$ and $N_{3 / 2}^{\mathrm{c}}( \pm 10 \%)$ are mainly due to the error in vapour-column length determination. Since the $N_{3 \mathrm{~S}}$ and $N_{3 / 2}^{\mathrm{c}}$ were measured along the same optical path, from relations (2) and (3) it follows that the ratio $N_{3 \mathrm{~S}} / N_{3 / 2}^{\mathrm{c}}$ appearing in equation (18) is determined with an error of $\pm 10 \%$. The intensity $I_{569}$ effectively diminished as the temperature grew, and therefore the accuracy of its determination accordingly decreased from $\pm 5 \%$ at the lowest, to $\pm 10 \%$ at the highest temperature. Namely, the number density in the $3 \mathrm{P}_{3 / 2}$ state was always maintained within the safe limits (regarding the trapping of the $4 \mathrm{D} \rightarrow 3 \mathrm{P}_{3 / 2}$ fluorescence), so that the 4D state was populated at similar rates at all investigated temperatures. On the other hand, the losses due to back-pooling and 4D-4F mixing increased proportionally to the increased $N_{3 \mathrm{~S}}$ density, causing the observed energy pooling signals to decrease rapidly. For this reason we have not extended measurements to even higher temperatures than reported. The lowest temperature that could be investigated with the present method $\left(567 \mathrm{~K}, N_{3 \mathrm{~S}}=2.2 \times 10^{14} \mathrm{~cm}^{-3}\right)$ was limited by the requirement to have pronounced quasistatic wing at detunings $\Delta \lambda \sim 0.1 \mathrm{~nm}$ (requirement $\Delta \lambda \ll \delta \lambda=0.01 \mathrm{~nm}$, see the text following equation (14)). Since the wing intensity $I_{3 / 2} \sim N_{3 \mathrm{~S}} N_{3 / 2}$ (equations (13) and (14)) and $N_{3 / 2}$ was always $\sim 10^{10} \mathrm{~cm}^{-3}$, the quasistatic wings diminished proportionally with decreasing sodium ground-state density. For this reason it was not possible to investigate the temperatures as low as $\left(483 \mathrm{~K}, N_{3 \mathrm{~S}} \sim 10^{12} \mathrm{~cm}^{-3}\right)$ in the experiment of Allegrini et al (1983). The intensity $I_{3 / 2}$ was measured with a relative error of $\pm 5 \%$ ( $T \geqslant 634 \mathrm{~K})$ which increased up to $10 \%$ when going towards the lowest temperature.

To gain the statistics we performed several measurements at each investigated temperature, by exciting the sodium atoms at different detunings $\Delta v_{L}$ in the wing, while keeping all other parameters nominally the same. The results obtained for $\sigma_{4 \mathrm{D}}$ by solving equation (18) for 


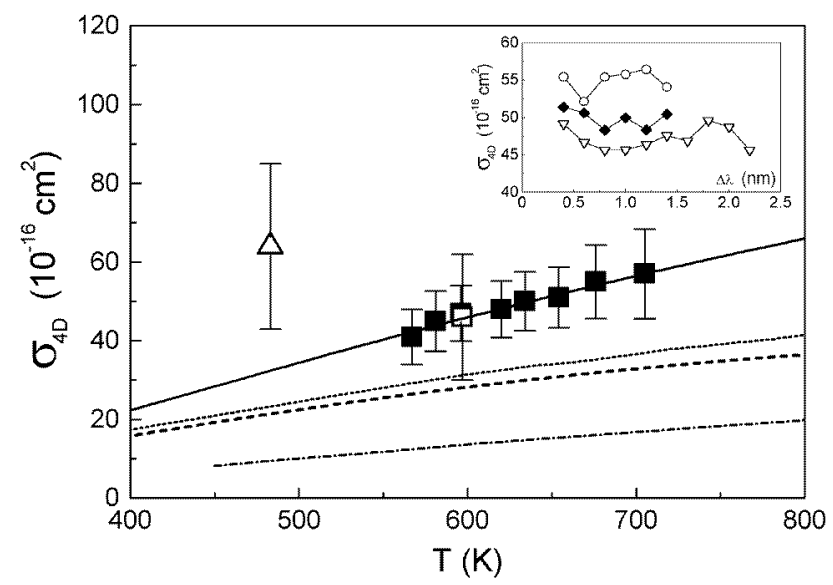

Figure 7. The cross section $\sigma_{4 \mathrm{D}}$ for the energy pooling process $\mathrm{Na}(3 \mathrm{P})+\mathrm{Na}(3 \mathrm{P}) \rightarrow \mathrm{Na}(4 \mathrm{D})+$ $\mathrm{Na}(3 \mathrm{~S})$, as a function of temperature. Experimental data are labelled with symbols: $\triangle$-Allegrini et al (1983), $\square-$ Huennekens and Gallagher (1983), $\square$-present work. Theoretical calculations are represented with curves (data are digitized from the figures in the original papers, except for Kowalczyk (1984) who tabulated the values): dot-Yurova et al (1994), dash-Geltman (1989), dash-dot-Kowalczyk (1984). The full curve is the Arrhenius-type fit $\sigma_{4 \mathrm{D}} \sim \sigma_{\infty} \exp (-\Delta E / k T)$, through the present experimental data. The inset shows the results for the $\sigma_{4 \mathrm{D}}$ obtained by solving equation (18) for a series of $I_{3 / 2}$ along the wing of the D2 line. Measurements were conducted at $N_{3 \mathrm{~S}}=3.3 \times 10^{15} \mathrm{~cm}^{-3}(T=654 \mathrm{~K})$, for three different $3 \mathrm{P}_{3 / 2}$ number densities realized by exciting the sodium atoms at different detunings $\Delta v_{L}$ in the wing: $-N_{3 \mathrm{P}_{3 / 2}}^{\mathrm{c}}=4 \times 10^{10} \mathrm{~cm}^{-3}$, $\mathrm{O}-N_{3 \mathrm{P}_{3 / 2}}^{\mathrm{c}}=5.6 \times 10^{10} \mathrm{~cm}^{-3}, \nabla-N_{3 \mathrm{P}_{3 / 2}}^{\mathrm{c}}=1.4 \times 10^{11} \mathrm{~cm}^{-3}$.

series of $I_{3 / 2}$ along the wing of the D2 line, for three measurements with different $N_{3 / 2}^{\mathrm{c}}$, are displayed in the inset of figure 7 . The scattering of the data along the wing detuning $\Delta \lambda$ is within $\pm 5 \%$ and reflects the uncertainty in the $I_{3 / 2}$ readings. No systematic trend with respect to the value of $N_{3 / 2}^{\mathrm{c}}$ is observed. The mean $\sigma_{4 \mathrm{D}}$ values for the displayed results are within a margin of at most $\pm 10 \%$, which reflects the influence of the dynamical conditions of the experiment (possible slight temperature and pump power fluctuations) on the results. With all sources of the uncertainties taken into account by adding errors in quadrature, the $k_{4 \mathrm{D}}$ was determined with an overall standard error between $\pm 15 \%$ (middle temperatures) and $20 \%$ (lowest and highest $T$ ).

The results for the energy pooling cross section $\sigma_{4 \mathrm{D}}$ in dependence on temperature are shown in figure 7, together with the previous experimental data of Allegrini et al (1983) and Huennekens and Gallagher (1983). The results of the theoretical calculations (Yurova et al 1994, Geltman 1989, Kowalczyk 1984) are also displayed.

Our data show that cross section $\sigma_{4 \mathrm{D}}$ slowly rises with the increased temperature, as expected for the endothermic $\mathrm{Na}(3 \mathrm{P})+\mathrm{Na}(3 \mathrm{P}) \rightarrow \mathrm{Na}(4 \mathrm{D})+\mathrm{Na}(3 \mathrm{~S})$ reaction. By fitting the $\ln \sigma_{4 \mathrm{D}} \sim 1 / T$ to the straight line we found that the cross section follows $\sigma_{4 \mathrm{D}} \sim \exp (-\Delta E / k T)$ dependence with $\Delta E=(608 \pm 95) \mathrm{cm}^{-1}$, which almost coincides with the energy defect $\left(613 \mathrm{~cm}^{-1}\right)$ for the considered process. The declared error bar includes the statistical uncertainty of the straight line fit through the data weighted by their error bars and uncertainty in determination of the abscissa $(T)$ value. The obtained value is in fair agreement with the values obtained by fitting the theoretical results to the same dependence. Namely, the $\sigma_{4 \mathrm{D}}(T)$ curves of Yurova et al (1994), Geltman (1989) and Kowalczyk (1984) yield $\Delta E$ values of 487 , 475 and $632 \mathrm{~cm}^{-1}$, respectively. As for the absolute values of $\sigma_{4 \mathrm{D}}$, our experimental data are 
higher than all theoretical calculations. The result closest to ours (Yurova et al 1994) is smaller by a factor of 1.5 .

\section{Discussion}

Prior to discussing our results in relation to previous experimental and theoretical findings, we are going to examine to what extent the presented results are influenced by the processes considered (4D $\leftrightarrow 4 \mathrm{~F}$ mixing, back pooling) in the evaluation procedure. If we had neglected $4 \mathrm{D} \leftrightarrow 4 \mathrm{~F}$ mixing the obtained values for $\sigma_{4 \mathrm{D}}$ would have been between $8 \%$ and $20 \%$ lower than displayed, the reduction rising progressively with the increased temperature. However, the $\sigma_{4 \mathrm{D}}$ would still exhibit mild temperature rise. On the other hand, if the $4 \mathrm{D} \leftrightarrow 4 \mathrm{~F}$ mixing is accounted for, but we disregard the back-pooling processes, the effect on the results is substantial. Namely, only the two points at the lowest temperatures remain practically unchanged, while in the rest of the interval the resulting $\sigma_{4 \mathrm{D}}$ values decrease from $42 \times 10^{-16} \mathrm{~cm}^{2}\left(T=581 \mathrm{~K}, N_{3 \mathrm{~S}}=3.5 \times 10^{14} \mathrm{~cm}^{-3}\right)$ to $28 \times 10^{-16} \mathrm{~cm}^{2}(T=705 \mathrm{~K}$, $N_{3 \mathrm{~S}}=1.1 \times 10^{16} \mathrm{~cm}^{-3}$ ), almost linearly with the temperature increase. This shows that under the present experimental conditions it was crucial to consider the back pooling in order to determine the cross section properly. For illustration, we mention that at the highest investigated temperature $(705 \mathrm{~K})$ the collisional rates are $R_{4 \mathrm{D}}^{\text {back }}=2.2 \times 10^{7} \mathrm{~s}^{-1}$, $C=5.8 \times 10^{7} \mathrm{~s}^{-1}$, while the spontaneous emission rate of the 4D level is $A=1.98 \times 10^{7} \mathrm{~s}^{-1}$, showing that the population and the depopulation of the 4D level is entirely dominated by collisional processes. Therefore, especially when endothermic (forward) energy pooling reactions are investigated at high ground-state densities, the role of the back-pooling processes should be carefully assessed, since the particular combination of the energy defect and the ratio of the statistical weights of the initial and final energy pooling state, could cause a pronounced effect on the results.

In our rate equations we have not considered the quenching of the $4 \mathrm{D}$ and $4 \mathrm{~F}$ levels. To our knowledge there are no data in the literature concerning the quenching of these states by collisions with the sodium ground-state atoms and molecules. However, the measurements $(T \sim 740 \mathrm{~K}$ ) of the various quenching processes of the $\mathrm{Na}(3 \mathrm{P})$ state in collisions with $\mathrm{Na}$ ground-state atoms and molecules were reported by Lam et al (1978). A strong molecular $\left(\sigma_{\text {mol }} \sim 10^{-14} \mathrm{~cm}^{2}\right)$ and weak atomic $\left(\sigma_{\mathrm{at}} \sim 10^{-17} \mathrm{~cm}^{2}\right)$ quenching were found to exist. A similar result was observed (Sasso et al 1992, Movre et al 1999) for the resonance state in caesium. The opposite has been found for the Cs(5D) state (Sasso et al 1992, Movre et al 1999): significant atomic $\left(\sigma_{\text {at }} \sim 10^{-15} \mathrm{~cm}^{2}, T \sim 600 \mathrm{~K}\right)$ and no molecular quenching have been observed. If we imagine the worst-case scenario and presume that both atomic (with $\sigma_{\text {at }}$ as high as for $\mathrm{Cs}(5 \mathrm{D})$ ) and molecular (of the same order of magnitude as for $\mathrm{Na}(3 \mathrm{P})$ ) quenching of the $\mathrm{Na}(4 \mathrm{D})$ and $\mathrm{Na}(4 \mathrm{~F})$ states are present, and include the quenching rates in the rate equations (5), (6), the resulting $\sigma_{4 \mathrm{D}}$ cross sections would be higher (from $2 \%$ to $10 \%$ as temperature increases from $634 \mathrm{~K}$ to $705 \mathrm{~K}$; at lower temperatures the influence is negligible), but still within the experimental error bars. In estimating the influence of quenching we have used the sodium dimer-atom ratio reported by Lam et al (1978).

Next, we will explain the choice of the particular value of $\beta=k_{4 \mathrm{~F}} / k_{4 \mathrm{D}}$ that was used in the evaluation of the results. Davidson et al (1986) reported the measurements (at $T=640 \mathrm{~K}$ ) of the ratio of the rate coefficients for producing $5 \mathrm{~S}_{1 / 2}, 4 \mathrm{D}_{3 / 2}, 4 \mathrm{D}_{5 / 2}, 4 \mathrm{~F}_{5 / 2}$ and $4 \mathrm{~F}_{7 / 2}$ states from the different combinations of $\mathrm{Na}\left(3 \mathrm{P}_{J}\right)+\mathrm{Na}\left(3 \mathrm{P}_{J^{\prime}}\right)$ collisions. They showed that the ratios are very sensitive to the particular $\left(J, J^{\prime}\right)=(1 / 2,1 / 2),(1 / 2,3 / 2),(3 / 2,3 / 2)$ combination actually involved in the collisions. For the ratio $k_{4 \mathrm{~F}} / k_{4 \mathrm{D}}$ that is of interest here $\left(k_{4 \mathrm{~F}}=k_{4 \mathrm{~F}_{5 / 2}}+k_{4 \mathrm{~F}_{7 / 2}}, k_{4 \mathrm{D}}=k_{4 \mathrm{D}_{3 / 2}}+k_{4 \mathrm{D}_{5 / 2}}\right)$ it was found that it changed from $0.38 \pm 0.06$ to 
$0.74 \pm 0.11$ when changing the collision regime from pure $(3 / 2,3 / 2)$ to pure $(1 / 2,1 / 2)$ collisions. When the fine-structure levels of the 3P states were populated in a nearly statistical ratio and all three combinations contributed to the energy pooling, the ratio $k_{4 \mathrm{~F}} / k_{4 \mathrm{D}}=0.55 \pm 0.05$ was found. We used this latter value in the evaluation since it was obtained under experimental conditions that coincide with ours regarding the temperature and the $N_{3 / 2} / N_{1 / 2}$ population ratio. For completeness we mention that there is another experimental result for the $k_{4 \mathrm{~F}} / k_{4 \mathrm{D}}$ ratio reported by Allegrini et al (1985). They excited the $3 \mathrm{P}_{3 / 2}$ level at low sodium densities $\left(N_{3 \mathrm{~S}} \approx 10^{13} \mathrm{~cm}^{-3}, T \sim 520 \mathrm{~K}\right.$ ) so that the excited-state population created was essentially pure $3 \mathrm{P}_{3 / 2}$. For that reason their value $k_{4 \mathrm{~F}} / k_{4 \mathrm{D}}=0.18 \pm 0.09$ is inadequate for use under our experimental conditions of near-statistical population of the 3P fine-structure levels.

The theoretical value of the $k_{4 \mathrm{~F}} / k_{4 \mathrm{D}}$ is available from the data of Yurova et al (1994) which yield $k_{4 \mathrm{~F}} / k_{4 \mathrm{D}}=0.13-0.14$ in the temperature range $600-700 \mathrm{~K}$. We have not used this theoretical value either, since the calculations disregard the role of the spin-orbit coupling.

The two previous experimental results (Allegrini et al 1983, Huennekens and Gallagher 1983) appear contradictory in the sense that $\sigma_{4 \mathrm{D}}$ tends to decrease with increasing $T$, which cannot be the case. Considering the original papers alone, one could not be conclusive about whether the value of Allegrini et al (1983) is too high, or the value of Huennekens and Gallagher (1983) is too low. As can be seen in figure 7, the cross section value $\sigma_{4 \mathrm{D}}$ measured by Huennekens and Gallagher (1983) is in excellent agreement with our experiment. The value of Allegrini et al (1983) is off-lying compared with all presented results, both experimental and theoretical.

In the experiment of Huennekens and Gallagher (1983) the quantities relevant for the evaluation of the cross section (sodium ground-state and 3P densities, spatial distribution and effective lifetime of the 3P state) were determined directly in the experiment (see also Huennekens 1982), and very little was left to rely upon the theoretical predictions. Moreover, the measured data are listed numerically so that subsequent assessment of the experiment is possible if desired. Only the effects of the trapping of the upper fluorescence (4D $\rightarrow 3 \mathrm{P}$ ) were accounted for by applying Holstein theory, and even that has a little impact on the reported $\sigma_{4 \mathrm{D}}$ value since it was obtained considering only those measurements performed in the conditions where the $4 \mathrm{D} \rightarrow 3 \mathrm{P}$ radiation trapping could have influenced the result by a few per cent at most. The experiment was seriously conducted, the sources of the errors were meticulously accounted for and the reported $\sigma_{4 \mathrm{D}}$ value should be considered reliable within the declared, fairly conservative, overall uncertainty.

More criticism can be addressed at the experiment of Allegrini et al (1983). The authors stated that the measurements of the effective lifetime $\left(\tau_{\text {eff }}\right)$ of the resonance level is complex and potentially troublesome and that they therefore avoided measuring $\tau_{\text {eff }}$ directly. In fact, they have not measured $\tau_{\text {eff }}$ either directly or indirectly. However, to obtain the cross section value from their measured quantity, eventually the value of the $\tau_{\text {eff }}$ had to be used explicitly. At that stage of the evaluation they used $\tau_{\text {eff }}$ calculated using Milne's theory (Milne 1926). As explained in Allegrini et al (1983) the general equation of Milne was solved for an optical depth $d=0.35 \mathrm{~mm}$, which the authors claimed was consistent with the geometrical configuration of their experiment. The measurements were performed in a capillary cell (diameter: $1.8 \mathrm{~mm}$ ) and the exciting laser beam was focused to the diameter of the cell window so as to illuminate the whole cross section of the cell. The fluorescence emerging from a disc $(1.8 \mathrm{~mm}$ in diameter and $150 \mu \mathrm{m}$ thick) adjacent to the entrance window was observed side-on. Allegrini et al (1983) gave no details on how the equivalent optical depth of $0.35 \mathrm{~mm}$ was determined. The term consistent, associated with this particular $d$ value, is rather non-committal, suggesting that some degree of arbitrariness could be present in its determination. Just to illustrate how sensitive this issue is, we mention that for an optical depth of $0.5 \mathrm{~mm}$ (the term consistent is 
flexible enough to apply to this value as well), the Milne theory would yield about $40 \%$ longer effective lifetime and consequently cross section $\sigma_{4 \mathrm{D}}$ smaller by a factor of two than actually reported.

Theoretical predictions for the energy dependence of this cross section are acceptably close to our experimental findings. In particular, the $\Delta E$ value stemming from the calculations of Kowalczyk (1984) is 4\% higher, while those resulting from calculations of Yurova et al (1994) and Geltman (1989) are about 30\% lower. Regarding the cross section absolute value, our data lie above the theoretical curves. Considering the data of Kowalczyk (1984) and the results of Geltman (1989) and Yurova et al (1994), the differences amount to a factor of 3 and 1.5, respectively. The accurate knowledge of the molecular potentials involved in the particular energy transfer process is essential for obtaining correct cross section values. However, we believe that in the present case, the causes for the disagreement between the experiment and the theory should not be sought on the part of poor knowledge of molecular potential curves, since the latest calculations performed by Yurova et al (1994) are based on the very accurate ab initio potentials. Other possible causes for the computed cross sections being systematically smaller than the measured ones are discussed in detail in the latest theoretical paper (Yurova et al 1994) and will not be repeated here. In brief, they all concern various effects that unavoidably influence the experimental values, but as yet have not been included in the theoretical treatment. We believe that accounting for the spin-orbit coupling would contribute a great deal to the improved agreement. The experiments (Davidson et al 1986, Vadla 1998, Jabbour et al 1996) have clearly demonstrated that the measured cross sections are substantially influenced by the actual relative population of the fine-structure sublevels of the initial state. Since these fine-structure effects are inherent to any experimental result, it is necessary to refine theoretical treatment in that regard, so as to enable the comparison of experiment and theory on equal grounds.

\section{Conclusion}

We have presented the measurements of the temperature dependence of the cross section $\sigma_{4 \mathrm{D}}$ for the energy pooling process $\mathrm{Na}(3 \mathrm{P})+\mathrm{Na}(3 \mathrm{P}) \rightarrow \mathrm{Na}(4 \mathrm{D})+\mathrm{Na}(3 \mathrm{~S})$, covering the range $567-705 \mathrm{~K}$. It has been found that the cross section increases with increasing temperature, following the $\sigma_{4 \mathrm{D}} \sim \exp (-\Delta E / k T)$ dependence. The value $\Delta E=(608 \pm 95) \mathrm{cm}^{-1}$, determined from the fit through the experimental data is in very good agreement with the energy defect $\left(613 \mathrm{~cm}^{-1}\right)$ for the considered process. The results reported here help to resolve the apparent contradiction between the two previous experimental results (Allegrini et al 1983, Huennekens and Gallagher 1983) which suggested that the $\sigma_{4 \mathrm{D}}$ tends to decrease with increasing temperature. Our measurements confirm the value of Huennekens and Gallagher (1983). The value reported by Allegrini et al (1983) is markedly too high compared with all previous (both experimental and theoretical) as well as the present results.

As for the absolute value, our experimental cross sections are higher than theoretical predictions (Kowalczyk 1984, Geltman 1989, Yurova et al 1994). The best agreement (factor of 1.5 difference) exists with the calculations of Yurova et al (1994), which are at the same time expected to be more reliable than others since they used accurate $a b$ initio molecular potentials to obtain the cross sections. We believe that agreement might be improved by refining the theoretical treatment. This concerns primarily the inclusion of fine-structure effects which have been proven to considerably affect the cross section values determined experimentally. 


\section{Acknowledgment}

We acknowledge financial support by the Ministry of Science and Technology, Republic of Croatia.

\section{Appendix}

The absorption coefficient in the quasistatic wings of alkali first resonance lines can be expressed in the form (Movre and Pichler 1980):

$$
k(Y)=\frac{e^{4}}{24 m^{2} c v_{0}\left(\Delta v_{\mathrm{fs}}\right)^{2}} f_{3 / 2}^{2} N_{0}^{2} \mathcal{K}(Y)
$$

where $Y=\Delta v / \Delta v_{\mathrm{fs}}, \Delta v$ is the frequency detuning in the wing measured from the centre of the line, $\Delta v_{\mathrm{fs}}$ is the fine-structure splitting, $v_{0}$ is the central frequency, $f_{3 / 2}$ is the oscillator strength of the D2 line, $N_{0}$ is the ground-state number density, and $\mathcal{K}(Y)$ is the reduced absorption coefficient. The remaining labels have their usual meaning. Here, we have rewritten the original expression (relation (16) in the original paper) using notation more convenient for evaluation of the experimental data.

Previously (Movre and Pichler 1980), the numerical $\mathcal{K}(Y)$ values were given for $Y$ up to 0.45 , while the reported analytical expressions were valid only for $Y \leqslant 0.3$. At higher temperatures in the present experiment the region of the quasistatic wing appropriate for the evaluation extended beyond one-third of the $3 \mathrm{P}$ fine-structure splitting, and it was therefore necessary to take into consideration the theoretical absorption profile at detunings farther in the wing.

Here, we have extended the $Y$ range, and the numerical values for the $\mathcal{K}(Y)$ in the red wing of the D1 and the blue wing of the D2 line are given in tables A1 and A2, respectively, for $Y \leqslant 3$. The quantities $\mathcal{P}_{J}(Y)=\mathcal{K}(Y) Y^{2}(J=1 / 2,3 / 2)$ were fitted to polynomial expressions and it has been found that the best reproduction of the numerical profiles is obtained by the following dependences:

$\mathcal{P}_{1 / 2}(Y)=1.0317+2.2934 \cdot Y-6.319 \times 10^{-1} \cdot Y^{2}+7.32 \times 10^{-2} \cdot Y^{3}$

Table A1. The reduced absorption coefficient $\mathcal{K}(Y)$ for the red wing of the self-broadened D1 resonance line of alkali metals. The reduced detuning $Y=\Delta v / \Delta v_{\mathrm{fs}}$ is measured from the D1 line centre.

\begin{tabular}{lcllllllll}
\hline$Y$ & $\mathcal{K}(Y)$ & $Y$ & $\mathcal{K}(Y)$ & $Y$ & $\mathcal{K}(Y)$ & $Y$ & $\mathcal{K}(Y)$ & $Y$ & $\mathcal{K}(Y)$ \\
\hline 0.01 & 10550 & 0.65 & 5.398 & 1.3 & 1.836 & 1.95 & 0.9578 & 2.6 & 0.594 \\
0.05 & 459.2 & 0.7 & 4.812 & 1.35 & 1.73 & 2 & 0.9189 & 2.65 & 0.5752 \\
0.1 & 125.3 & 0.75 & 4.325 & 1.4 & 1.633 & 2.05 & 0.8824 & 2.7 & 0.5573 \\
0.15 & 60.33 & 0.8 & 3.914 & 1.45 & 1.545 & 2.1 & 0.848 & 2.75 & 0.5403 \\
0.2 & 36.51 & 0.85 & 3.563 & 1.5 & 1.463 & 2.15 & 0.8157 & 2.8 & 0.524 \\
0.25 & 24.99 & 0.9 & 3.261 & 1.55 & 1.389 & 2.2 & 0.7853 & 2.85 & 0.5085 \\
0.3 & 18.45 & 0.95 & 2.999 & 1.6 & 1.32 & 2.25 & 0.7566 & 2.9 & 0.4936 \\
0.35 & 14.35 & 1 & 2.769 & 1.65 & 1.256 & 2.3 & 0.7294 & 2.95 & 0.4794 \\
0.4 & 11.57 & 1.05 & 2.567 & 1.7 & 1.197 & 2.35 & 0.7037 & 3 & 0.4658 \\
0.45 & 9.597 & 1.1 & 2.387 & 1.75 & 1.142 & 2.4 & 0.6794 & & \\
0.5 & 8.128 & 1.15 & 2.227 & 1.8 & 1.091 & 2.45 & 0.6564 & & \\
0.55 & 7 & 1.2 & 2.083 & 1.85 & 1.044 & 2.5 & 0.6345 & & \\
0.6 & 6.113 & 1.25 & 1.954 & 1.9 & 0.9993 & 2.55 & 0.6138 & & \\
\hline
\end{tabular}


Table A2. The reduced absorption coefficient $\mathcal{K}(Y)$ for the blue wing of the self-broadened D2 resonance line of alkali metals. The reduced detuning $Y=\Delta v / \Delta v_{\mathrm{fs}}$ is measured from the D2 line centre.

\begin{tabular}{lcllllllll}
\hline$Y$ & $\mathcal{K}(Y)$ & $Y$ & $\mathcal{K}(Y)$ & $Y$ & $\mathcal{K}(Y)$ & $Y$ & $\mathcal{K}(Y)$ & $Y$ & $\mathcal{K}(Y)$ \\
\hline 0.01 & 30850 & 0.65 & 9.406 & 1.3 & 2.643 & 1.95 & 1.254 & 2.6 & 0.7356 \\
0.05 & 1264 & 0.7 & 8.209 & 1.35 & 2.466 & 2 & 1.197 & 2.65 & 0.7099 \\
0.1 & 325.2 & 0.75 & 7.233 & 1.4 & 2.307 & 2.05 & 1.143 & 2.7 & 0.6856 \\
0.15 & 148.3 & 0.8 & 6.426 & 1.45 & 2.163 & 2.1 & 1.094 & 2.75 & 0.6625 \\
0.2 & 85.41 & 0.85 & 5.751 & 1.5 & 2.033 & 2.15 & 1.047 & 2.8 & 0.6406 \\
0.25 & 55.87 & 0.9 & 5.179 & 1.55 & 1.914 & 2.2 & 1.003 & 2.85 & 0.6198 \\
0.3 & 39.59 & 0.95 & 4.692 & 1.6 & 1.805 & 2.25 & 0.9624 & 2.9 & 0.6 \\
0.35 & 29.64 & 1 & 4.271 & 1.65 & 1.706 & 2.3 & 0.9239 & 2.95 & 0.5811 \\
0.4 & 23.1 & 1.05 & 3.907 & 1.7 & 1.615 & 2.35 & 0.8878 & 3 & 0.5631 \\
0.45 & 18.55 & 1.1 & 3.588 & 1.75 & 1.531 & 2.4 & 0.8537 & & \\
0.5 & 15.26 & 1.15 & 3.308 & 1.8 & 1.454 & 2.45 & 0.8216 & & \\
0.55 & 12.8 & 1.2 & 3.06 & 1.85 & 1.382 & 2.5 & 0.7913 & & \\
0.6 & 10.9 & 1.25 & 2.839 & 1.9 & 1.316 & 2.55 & 0.7627 & & \\
\hline
\end{tabular}

valid for the red wing of the D1 line, and

$$
\begin{gathered}
\mathcal{P}_{3 / 2}(Y)=3.0752+1.8476 \cdot Y-8.508 \times 10^{-1} \cdot Y^{2}+2.278 \times 10^{-1} \cdot Y^{3} \\
-2.53 \times 10^{-2} \cdot Y^{4}
\end{gathered}
$$

which holds for the blue wing of the D2 line. The relations (A2) and (A3) reproduce the numerical profiles with an accuracy better than $\pm 0.5 \%$ in the whole $Y$ range.

With expressions (A2) and (A3) taken into account, from equation (A1) we obtain the following expressions for the absorption coefficient in the outer wings of the alkali first resonance lines:

$$
\begin{aligned}
& k_{1 / 2}(\Delta v)=\frac{e^{4}}{24 m^{2} c v_{1 / 2}} f_{3 / 2}^{2} N_{0}^{2} \frac{\mathcal{P}_{1 / 2}(Y)}{(\Delta v)^{2}} \\
& k_{3 / 2}(\Delta v)=\frac{e^{4}}{24 m^{2} c v_{3 / 2}} f_{3 / 2}^{2} N_{0}^{2} \frac{\mathcal{P}_{3 / 2}(Y)}{(\Delta v)^{2}}
\end{aligned}
$$

that are valid for the detunings in the wing $\Delta v \leqslant 3 \times \Delta v_{\mathrm{fs}}$.

\section{References}

Allegrini M, Alzetta G, Kopystynska A and Moi L 1976 Opt. Commun. 1996 Allegrini M, Bicchi P and Moi L 1983 Phys. Rev. A 281338

Allegrini M, Gabbanini C, Moi L and Colle R 1985 Phys. Rev. A 322068

Bezuglov N N, Klucharev A N and Sheverev V A M 1987 J. Phys. B: At. Mol. Opt. Phys. 202497

Davidson S A, Kelly J F and Gallagher A 1986 Phys. Rev. A 333756

Demtröder W 1996 Laser Spectroscopy (Berlin: Springer) pp 896-901

Geltman S 1989 Phys. Rev. A 402301

Horvatic V, Movre M, Beuc R and Vadla C 1993 J. Phys. B: At. Mol. Opt. Phys. 263679

Huennekens J P 1982 PhD Thesis University of Colorado

Huennekens J and Gallagher A 1983 Phys. Rev. A 27771

Jabbour Z J, Namiotka R K, Huennekens J, Allegrini M, Milosevic S and de Tomasi F 1996 Phys. Rev. A 541372

Kowalczyk P 1984 J. Phys. B: At. Mol. Phys. 17817

Krause L 1975 The Excited State in Chemical Physics ed J W McGowan (New York: Wiley) pp 267-316

Krebs D J and Schearer L D 1980 J. Chem. Phys. 753340

Kushawaha V S and Levental J J 1982 Phys. Rev. A 25570

Lam L K, Fujimoto T and Gallagher A C 1978 J. Chem. Phys. 683553 
Magnier S, Aubret-Frécon M, Bouty O, Masnou-Seeuws F, Millié Ph and Ostrovskii V N 1994 J. Phys. B: At. Mol. Opt. Phys. 271723

Magnier S, Millié Ph, Dulieu O and Masnou-Seeuws F 1993 J. Chem. Phys. 987113

Milne E A 1926 J. London Math. Soc. 140

Movre M, Horvatic V and Vadla C 1999 J. Phys. B: At. Mol. Opt. Phys. 324647

Movre M and Pichler G 1980 J. Phys. B: At. Mol. Phys. 13697

Philipsen P H T, Nijland J H, Rudolph H and Heideman H G M 1993 J. Phys. B: At. Mol. Opt. Phys. 26939

Sasso A, Demtröder W, Colbert T, Wang C, Ehrlacher E and Huennekens J 1992 Phys. Rev. A 451670

Scholz T, Schiffer M, Welzel J, Cysarz D and Lange W 1996 Phys. Rev. A 532169

Vadla C 1998 Eur. Phys. J. D 1259

Vadla C, Niemax K and Brust J 1996 Z. Phys. D 37241

Vadla C, Niemax K and Horvatic V 1998 Eur. Phys. J. D 1139

Yurova I Yu, Dulieu O, Magnier S, Masnou-Seeuws F and Ostrovskii V N 1994 J. Phys. B: At. Mol. Opt. Phys. 27 3659

Wiese W L, Smith M W and Miles B M 1969 Atomic Transition Probabilities (NSRDS-NBS 22) vol 2 (Washington DC: US Govt Printing Office) 